\title{
DIFFERENTIAL CRITERIA FOR FLATNESS
}

\author{
SARAH GLAZ
}

\begin{abstract}
We introduce differential algebra methods to the study of flatness over Noetherian domains. The results concern the specific cases of ideals and attempt to use the underlying divisibility properties of the ring. They concern mostly regular (geometric) rings and one-dimensional rings.
\end{abstract}

Introduction. The purpose of this paper is to introduce new criteria for flatness of ideals in commutative, Noetherian rings. The methods generally used in the literature are homological in nature. We introduce differential conditions which will imply flatness of ideals in certain types of rings.

Let $A$ be a Noetherian ring, and let $\underline{d}: A \rightarrow M$ be a derivation from $A$ to an $A$-module $M$. For two elements $f$ and $g$ in an ideal $I$ of $A$, let $\Delta_{d}(f, g)=f \underline{d}(g)-$ $g \underline{d}(f)$. Then $\Delta_{\underline{d}}(f, g) \in I M$, and, if $I$ is a flat ideal of $A, \Delta_{d}(f, g) \in I^{2} M$ [7].

We introduce the following conditions:

(D1) For each $A$-module $M$, every derivation $\underline{d}: A \rightarrow M$ and any two elements $f$ and $g$ in $I, \Delta_{d}(f, g) \in I^{2} M$. $I^{2}$.

(D2) For every derivation $\underline{d}: A \rightarrow A$ and any two elements $f$ and $g$ in $I, \Delta_{\underline{d}}(f, g) \in$

We investigate to what extent condition (D1) imposed on an ideal $I$, implies its flatness and in which types of rings conditions (D2) will suffice.

Let $\left(\Omega_{A / Z}, D\right)$ be the module of Kähler differentials of $A$ viewed as an algebra over the integers $Z, \Omega_{A / Z}$, with the canonical derivation $D: A \rightarrow \Omega_{A / Z}$, and let $I, f$ and $g$ be as above. The universal property of $\left(\Omega_{A / Z}, D\right)$ ensures that $\Delta_{d}(f, g) \in$ $I^{2} M$ for every derivation $\underline{d}$ into an $A$-module $M$ iff $\Delta_{\underline{D}}(f, g) \in I^{2} \Omega_{A / Z}$; hence the problem could be discussed using the $A$-module $\Omega_{A / Z}$ only. Unfortunately, one does not have sufficient information about the module $\Omega_{A / Z}$, even in cases where the ring $A$ is relatively simple, to pursue an investigation along this line. It is more practical to use other modules, e.g. $A$ itself, as in condition (D2), or various completions of over-rings of $A$.

Both differential conditions and the flatness property can be checked locally, hence in the following we will let $A$ be a local ring. Furthermore, to eliminate trivial cases, we shall require $A$ to be a domain.

The author wishes to thank Professor W. V. Vasconcelos for suggesting the problem and for many valuable discussions.

Presented to the Society January 6, 1978; received by the editors January 19, 1979, and, in revised form, May 22, 1979.

AMS (MOS) subject classifications (1970). Primary 13C10; Secondary $13 \mathrm{~B} 10$.

Key words and phrases. Regular ring, C. M. ring, affine domain, derivation, flat.

(c) 1980 American Mathematical Society 0002-9939/80/0000-0203/\$02.50 
1. Regular rings. Let $(A, \underline{m})$ be a regular local ring with maximal ideal $\underline{m}$, the characteristic of $A$ and its residue field equal to 0 . Let $x_{1}, \ldots, x_{n}$ be a regular system of parameters for $A$. The $\underline{m}$-adic completion of $A, \hat{A}$, contains a coefficient field $K$ of charateristic 0 . In fact $\hat{A}=K\left[\left[x_{1}, \ldots, x_{n}\right]\right]$, the power series ring in $x_{1}, \ldots, x_{n}$ over $K$, and $\operatorname{Der}_{K}(\hat{A})=\sum_{i=1}^{n} \hat{A} \partial / \partial x_{i}$, where $\partial / \partial x_{i}: \hat{A} \rightarrow \hat{A}$ denotes the partial derivation of $\hat{A}$ with respect to $x_{i}$, is a free module of rank $n$. If $A$ contains a field $k$ over which $A / \underline{m}$ is separably algebraic, then $k \subset K$ and $\operatorname{Der}_{k}(\hat{A})=$ $\operatorname{Der}_{K}(\hat{A})$.

Let $I$ be an ideal of $A$ satisfying (D1), then the ideal $I \hat{A}$ of $\hat{A}$ satisfies (D2).

TheOREM 1.1. Let $(A, \underline{m})$ be a regular local ring with $\mathrm{ch} A / \underline{m}=0$ and let $I$ be an ideal of $A$ satisfying (D1); then $I$ is flat.

Proof. We may assume $A=K\left[\left[x_{1}, \ldots, x_{n}\right]\right], K$ a field of characteristic 0 and $I$ satisfies (D2) and is not flat.

Consider first the case where $I$ is generated by two elements. Since $A$ is a unique factorization domain [4], we may assume they form a regular sequence in $I$, and thus $I / I^{2}$ is a free $A / I$-module [3]. This implies that the second exterior power of $I / I^{2}, \Lambda^{2}\left(I / I^{2}\right)$, is a free $A / I$-module. We will exhibit a nonzero element in $A / I$ which annihilates $\Lambda^{2}\left(I / I^{2}\right)$ thus leading to a contradiction.

Let $u, v$ and $w$ be elements of $I$, then $u(v \wedge w)=u(w \wedge v)$ and $2 u(v \wedge w)=0$. Thus $I$ annihilates $\Lambda^{2} I$. Note that this implies $\Lambda^{2} I=\Lambda^{2}\left(I / I^{2}\right)$. Next we note that the differential condition for $\underline{d}=\partial / \partial x_{i}$ implies that $\partial u / \partial x_{i}$ annihilates $\Lambda^{2} I$ for every element $u$ of $I$. Indeed,

$$
\begin{aligned}
\frac{\partial}{\partial x_{i}} u(v \wedge w) & =\left(\frac{\partial}{\partial x_{i}} u\right) v \wedge w=\left(\frac{\partial}{\partial x_{i}} v\right) u \wedge w=u \wedge\left(\frac{\partial}{\partial x_{i}} v\right) w \\
& =u \wedge v\left(\frac{\partial}{\partial x_{i}} w\right)=u\left(\frac{\partial}{\partial x_{i}} w\right) \wedge v \\
& =\left(\frac{\partial}{\partial x_{i}} u\right) w \wedge v=\left(\frac{\partial}{\partial x_{i}} u\right) w \wedge v .
\end{aligned}
$$

Thus $\left(\partial u / \partial x_{i}\right) v \wedge w=0$ for $1 \leqslant i \leqslant n$.

For a power series $h$ in $A$ we write $h=h_{r}+H_{r}$ where $h_{r}$ is the homogeneous component of $h$ of minimal total degree $r$ in $x_{1}, \ldots, x_{n}$, and $H_{r}$ is a power series with homogeneous components of total degree higher than $r$. We call $r=\operatorname{deg}(h)$.

Let $u=u_{r}+U_{r}$ be a power series in $I$ of minimal degree $r>1$; then $\partial u / \partial x_{i} \notin$ $I$ for some $1 \leqslant i \leqslant n$, thus the class of $\partial u / \partial x_{i}$ in $A / I$ is nonzero.

Next consider the general case:

Let $u=u_{r}+U_{r}$ in $I$ as above. We call $r=\operatorname{deg}(I)$. We then have that $\operatorname{deg}\left(I^{2}\right)=$ $2 r$. Let $v=v_{r}+V_{r}$ be anther element of $I$ of degree $r$, then $\Delta_{\partial / \partial x_{i}}(u, v) \in I^{2}$, $\operatorname{deg} \Delta_{\partial / \partial x_{i}}(u, v) \geqslant 2 r-1$ and

$$
\Delta_{\partial / \partial x_{i}}(u, v)=\Delta_{\partial / \partial x_{i}}\left(u_{r}, v_{r}\right)+\Delta_{\partial / \partial x_{i}}\left(u_{r}, V_{r}\right)+\Delta_{\partial / \partial x_{i}}\left(U_{r}, v_{r}\right)+\Delta_{\partial / \partial x_{i}}\left(U_{r}, V_{r}\right) .
$$

We conclude that $\Delta_{\partial / \partial x_{i}}\left(u_{r}, v_{r}\right)=0$ for every $i$. Using the remark above we have that $v_{r}$ is a multiple of $u_{r}$ (by an invertible element of $A$ ). 
Let $v=v_{n}+V_{n}, n>r$, be an element of $I$ of degree $n$. If, for every integer $n>r, u_{r}$ divides $v_{n}$, then $I \subseteq(u)+\underline{m}^{i}$ for every $i$, which by Krull intersection theorem [4], implies $I=(u)$. Let $n>r$ be a minimal integer such that $u_{r}$ does not divide $v_{n}$.

We give a list of possible generators for $I$. The generators of $I$ of degree $r$ are of the form $u_{r}+U_{r}^{\prime}$. If $w=w_{s}+W_{s}$ is a generator of $I$ of degree $s<n$, $u_{r}$ divides $w_{s}$, hence by subtracting suitable multiples of $u$ from $w$, we can replace $w$ by a generator of degree higher than or equal to $n$. We then have a finite number of generators of degree higher than or equal to $n$.

We expand $\Delta_{\partial / \partial x_{i}}(u, v)$ as in formula (1) and set it equal to a summation of the generators of $I^{2}$ with coefficients in $A$. Since $u_{r}$ does not divide $v_{n}, \Delta_{\partial / \partial x_{i}}\left(u_{r}, v_{n}\right) \neq 0$ for some $i$, and $\operatorname{deg} \Delta_{\partial / \partial x_{i}}(u, v)=r+n-1$. Comparing components of minimal degree on both sides of the equality we obtain a power series $z$, such that $\Delta_{\partial / \partial x_{i}}\left(u_{r}, v_{n}\right)=z u_{r}^{2}$. Applying the result of the case where the ideal is generated by two elements we obtain an element $w=a u_{r}+b v_{n}$ with $a$ and $b$ in $A$ and $\operatorname{deg} w<r$, which generates the ideal generated by $u_{r}$ and $v_{n}$. But then the element $w+a U_{r}+b V_{n} \in I$ and is of degree lower than $r$.

The following example shows that if $(A, \underline{m})$ is a regular local ring of characteristic 0 , but ch $A / \underline{m}=p>0$, condition (D1) does not necessarily imply flatness. Let $A=Z_{p}[x]$, the polynomial ring in one variable over the integers localizd at a prime number $p$. Let $I$ be the ideal generated by $p$ and $x^{p}$, and let $\underline{d}: A \rightarrow M$ be a derivation (necessarily over $Z_{p}$ ) of $A$ into an $A$-module $M$; then $\Delta_{d}\left(p, x^{p}\right)=$ $p^{2} x^{p-1} \underline{d}(x)$. Thus $\Delta_{d}(f, g) \in I^{2} M$ for every $f$ and $g$ in $I$, but $I$ is not flat. This example points out the need of enough derivations of the ring, hence the need to restrict ourselves to equal characteristic rings.

Next we consider a subclass of the regular local rings for which condition (D2) suffices to imply flatness.

Following Matsumura [5] we define:

Definition. A regular local ring $(A, \underline{m})$ containing $a$ field $k$ is said to be of analytic type over $k$ if it satisfies:

(1) $A / \underline{m}$ is algebraic over $k$ and $\operatorname{ch}(k)=0$,

(ii) $\operatorname{rank} \operatorname{Der}_{k}(A)=$ Krull $\operatorname{dim} A$.

For such a ring $(A, \underline{m})$ we have that the derivations $\partial / \partial x_{i}$ of the $\underline{m}$-adic completion of $A, \operatorname{map} A$ into itself [5], hence we conclude:

TheOREM 1.2. Let $(A, \underline{m})$ be a regular local ring containing $a$ field $k$ and of analytic type over $k$, and let $I$ be an ideal of $A$ satisfying (D2), then I is flat.

2. One-dimensional rings. Let $k$ be a algebraically closed field of characteristic 0 , and let $(A, m)$ be a one-dimensional, local $k$-algebra essentially of finite type, with $A / \underline{m} \simeq k$. Assume $A$ is an analytically irreducible domain and let $\bar{A}$ denote the integral closure of $A$ in its quotient field. We have that every localization of $\bar{A}$ at a maximal ideal $\underline{n}$ of $\bar{A}, \bar{A}_{\underline{n}}$, is a discrete valuation ring with residue field isomorphic to $k$ (Krull-Akizuki theorem, [6]). Thus the $\underline{n}$-adic completion of $\overline{A_{\underline{n}}},\left(\frac{\hat{\bar{A}}}{\underline{n}}\right)$, is equal to $k\left[[x]\right.$, a power series over $k$, where $x$ is the uniformizing parameter of $\bar{A}_{\underline{n}}$ [2]. In 
this case we have an embedding of finite type $\hat{A} \hookrightarrow\left(\hat{\overline{A_{n}}}\right)[8]$. Let $x^{N} k[[x]]$ be the conductor ideal of $k[[x]]$ into $\hat{A}$; then $\hat{A} / x^{N} k[[x]]$ is generated as a vector space over $k$ by the classes of $\left\{1, h_{1}, \ldots, h_{k}\right\}$, where $h_{i}$ are power series in $k[[x]]$ with $n_{i}=\operatorname{deg} h_{i}=$ initial power of $x$ in $h_{i}$, strictly less than $N$. Thus every element of $\hat{A}$ can be written as a $k$-linear combination of $\left\{1, h_{1}, \ldots, h_{k}\right\}$ plus an element of $x^{N} k[[x]]$. We readjust the above set of power series so as to have the following properties:

(1) $1<n_{1}<n_{2}<\ldots<n_{k}<N$.

(2) If $n_{i}=j n_{1}$ for some $1 \leqslant i \leqslant k$ and some $j$, then $h_{i}=h_{1}^{j}$.

For a subset $S$ of $k[[x]]$ put $\nu(S)=\{n ; n=\operatorname{deg} f, f \in S\}$ and note that $\nu(\hat{A})=$ $\left\{0, n_{1}, \ldots, n_{k}, N, N+1, \ldots\right\}$.

Let $J$ be a nonflat ideal of $\hat{A}$ which satisfies (D1), and let $f$ be a nonzero element of $J$ of minimal degree $n>0$. Let $g$ be an element of $J$ of degree $m \neq n$ which is not a multiple of $f$. The Krull intersection theorem [4] allows us to pick $g$ such that $u=m-n \notin \nu(A)$; let $m$ be minimal satisfying this property. We then readjust a set of generators for $J$, by subtracting suitable multiples of $f$ from necessary elements, to consist of $f$ (necessarily in every minimal set of generators of $J$ ) and a finite number of elements of degree higher than or equal to $m$.

Let $\underline{d}: \hat{A} \rightarrow k[[x]]$ be the restriction of the derivation $\partial / \partial x$ of $k[[x]]$ to $\hat{A}$, and let $M$ be the $\hat{A}$-module $\hat{A} \underline{d}(\hat{A})$; then $\underline{d}: \hat{A} \rightarrow M$. Note that an element of $M$ can be written as a $k$-linear combination of $\left\{h_{i}^{\prime}(1 \leqslant i \leqslant k), h_{i} h_{j}^{\prime}(1<i, j<k)\right\}$ and a power series in $x^{N-1} k[[x]]$.

Next we consider $\Delta_{d}(f, g)$ an element of $J^{2} M$ of degree $n+m-1=2 n+$ $(u-1)$. Noting that a generating set for $J^{2}$ will consist of $f^{2}$ and elements of degree higher than or equal to $n+m$, we necessarily have $\Delta_{d}(f, g)=f^{2} \varepsilon+F$ where $\varepsilon \in M, \operatorname{deg} \varepsilon=u-1$ and $\operatorname{deg} F>n+m-1$.

Theorem 2.1. Let $(A, \underline{m})$ and $k$ be as in the above discussion, and let $I$ be an ideal of $A$ satisfying (D1). If $h_{i}$ are monomials for every $1 \leqslant i<k$, then $I$ is flat.

Proof. We may replace $A$ by $\hat{A}$ and thus assume $A$ has the structure described above and let $J=I \hat{A}$. In this case a given integer does not belong to $\nu(A)$ iff it does not occur as a power of $x$ in any element of $A$. Since $n_{1}>1$ and $u \notin \nu(A)$, $u-1$ does not occur as a power of $x$ in any element of $M$.

Proposition 2.2. Let $(A, \underline{m})$ and $k$ be as in the above discussion and let $I$ be an ideal of $A$ satisfying (D1). If $J=I \hat{A}$ satisfies $u<n_{1}$ or $n_{1}<u<n_{2}$ then $I$ is flat.

Proof. Assume $A=\hat{A}$ and $J=I$. The case where $u<n_{1}$ is clear. If $n_{1}<u<$ $n_{2}$, the only element of $M$ where $x^{u-1}$ can occur is of the form $\alpha_{1} h_{1}^{\prime}+\alpha_{2} h_{1} h_{1}^{\prime}+G$, where $\alpha_{1}, \alpha_{2} \in k$ and $\operatorname{deg} G \geqslant u$. Since $\operatorname{deg} h_{1}^{\prime}=n_{1}-1$ and $\operatorname{deg} h_{1} h_{1}^{\prime}=2 n_{1}-1$, $\alpha_{1}$ must be equal to 0 , but then $u-1$ cannot occur as an initial power.

Theorem 2.3. Let $(A, \underline{m})$ and $k$ be as in the above discussion, and let $I$ be a prime ideal of $A$ satisfying (D1), then $I$ is flat. 
Proof. We may assume $A$ is complete with the discussed structure and $I$ is the maximal ideal of $A, \underline{m}$. Let $f=h_{1}$ and $g=h_{i}$, $i$ minimal with the property that $u=n_{i}-n_{1} \notin \nu(A)$. For $l<i$ we have that $n_{l}-n_{1} \in \nu(A)$ and since $h_{l-1} h_{1} \in A$ for every $l \leqslant i$ we obtain $n_{l}-n_{1}<n_{l-1}$ for $l<i$ and $u=n_{i}-n_{1}<n_{i-1}$. Using the second property of the set $\left\{h_{1}, h_{2}, \ldots, h_{k}\right\}$ we obtain $h_{l}=h_{1}^{l}$ for $l<i$. As in our previous results we aim to show that an element of degree $u-1$ cannot be written as a $k$-linear combination of:

$$
\begin{aligned}
& h_{1}^{\prime}, h_{2}^{\prime}, \ldots, h_{k}^{\prime} \\
& h_{1} h_{1}^{\prime}, h_{1} h_{2}^{\prime}, \ldots, h_{1} h_{k}^{\prime} \\
& \vdots \\
& h_{k} h_{1}^{\prime}, h_{k} h_{2}^{\prime}, \ldots, h_{k} h_{k}^{\prime} \text { plus an element in } x^{N-1} k[[x]] .
\end{aligned}
$$

Assuming this is possible, let the coefficients of the first row be $\alpha_{1}, \alpha_{2}, \ldots, \alpha_{k}$, the coefficients of the second row be $\beta_{1}, \beta_{2}, \ldots, \beta_{k}$, etc. We have $\alpha_{1}=0 . h_{2}=h_{1}^{2}$ implies $h_{2}^{\prime}=2 h_{1} h_{1}^{\prime}$ and thus $2 \alpha_{2}+\beta_{1}=0$. Continuing the diagonalized annihilation (e.g. $h_{3}$ with $h_{1} h_{2}^{\prime}$ and $h_{2} h_{1}^{\prime}$ ) we obtain an initial power of degree higher than $n_{i-1}-1$.

Proposition 2.4. Let $(A, m)$ and $k$ as in Theorem 2.3, and let $I$ be an ideal of $A$ satisfying (D1) with $J=I \hat{A}$ generated by $h_{i}, s \leqslant i<k$ for some fixed $s>1$. Then $I$ is flat.

Proof. Put $f=h_{s}$ and $g=h_{i+s}$ some $i \geqslant 1, i+s$ minimal such that $n_{i+s}-n_{s} \notin$ $\nu(A)$. Note that $n_{l+s}-n_{s} \leqslant n_{l}$ for $l \leqslant i$ and $n_{i+s}-n_{s}<n_{i}$. Thus $n_{l}=\ln _{1}$ for $l<i$ and the proof follows.

The following example will show that even in the case where $h_{i}$ are monomials and $I$ is a prime ideal of $A$, condition (D2) will not suffice to imply flatness. Let $k$ be an algebraically closed field of characteristic $0 . A=k\left[\left[t^{2}, t^{3}\right]\right]$ and $I$ the ideal generated by $t^{2}$ and $t^{3}$. Let $\underline{d}: A \rightarrow A$ be a derivation over $k$. Then $\underline{d}\left(\left(t^{2}\right)^{3}\right)=$ $\underline{d}\left(\left(t^{3}\right)^{2}\right)$ and $3 t^{4} \underline{d}\left(t^{2}\right)=2 t^{3} \underline{d}\left(t^{3}\right)$ which imply $\underline{d}\left(t^{2}\right)$ and $\underline{d}\left(t^{3}\right)$ have no constant terms. Hence $\underline{d}(I) \subset I$ and $I$ satisfies (D2) without being flat.

A full solution of the one-dimensional case will be of value in extending the differential criteria of flatness to affine domains.

CONJECTURE. Let $k$ be a field of characteristic 0 , and let $(A, m)$ be a one-dimensional, local, essentially of finite type $k$-algebra, which is a domain. Let $I$ be an ideal of $A$ satisfying (D1); then I is flat.

Based on the validity of this conjecture we prove:

TheORem 2.5. Let $A$ be a Cohen Macaulay affine domain over a field of characteristic 0 , and let $I$ be an ideal of $A$ satisfying (D1); then $I$ is flat.

Proof. We may assume $A$ is local with maximal ideal $\underline{m}, \operatorname{Krull} \operatorname{dim} A=n$ and the theorem is true for lower dimensional Cohen Macaulay affine domains.

Let $p_{1}, \ldots, p_{n}$ be the prime ideals associated with $I$. By the induction hypotheses $\operatorname{ht}\left(p_{i}\right)=1$ or $p_{i}$ is equal to $\underline{m}$, hence $I=J \cap L$, where $J$ is an intersection of height 
one ideals associated with the $p_{i}$ 's which have height equal to one and $L=0$ or $L$ is an $\underline{m}$-primary ideal.

Construct the faithfully flat extension of $A, A(x)=A[x]_{\underline{m} A[x]^{*}}$.

Case (i). $I=J \cap L, L \neq 0$; let $a$ and $b$ be a regular sequence in $L \backslash \cup_{\mathrm{ht}\left(p_{i}\right)=1} p_{i}$ and $f=a^{2} x+b^{2}$. Note that $f \in \underline{m} L A(x)$ and that $(f)$ is a prime ideal of $A(x)$. By the induction hypothesis $I A(x)+f A(x) / f A(x) \simeq I A(x) / f A(x) \cap I A(x)$ is flat. By the choice of $f$ we have $f A(x) \cap J A(x)=f J A(x)$ and thus $f A(x) \cap I A(x) \subset$ $\underline{m} I A(x)$. We conclude that $I A(x) / \underline{m} I A(x)$ is flat and $I A(x)$ is flat.

Case (ii). $I=J$; pick $a$ in $I$ and $b$ in $\underline{m} \backslash\left(\left(\cup p_{i}\right) \cup\left(\cup q_{i}\right)\right)$ where $q_{i}$ run over the associated primes of $(a)$. Then $a$ and $b$ form a regular sequence and let $f=a x+b$. By the induction hypothesis $I A(x)+f A(x) / f A(x)$ is flat, generated, say, by the class of a polynomial $g$ in $I A(x)$. Since all the associated primes of $I$ have height equal to one we have that $I A(x) \subseteq(g)+(f)^{i}$ for every $i$, thus by the Krull Intersection theorem [4], $I A(x)=(g)$.

\section{REFERENCES}

1. N. Bourbaki, Commutative algebra, Addison-Wesley, Reading, Mass., 1972.

2. W. Fulton, Algebraic curves, Benjamin, New York, 1969.

3. I. Kaplansky, Commutative rings, Allyn and Bacon, Boston, Mass., 1970.

4. H. Matsumura, Commutative algebra, Benjamin, New York, 1970.

5. Formal power series rings over polynomial rings. I, Number Theory, Algebraic Geom. and Commutative Alg. in honor of Y. Akizuki, Kinokuniya, Tokyo, 1973, pp. 521-528.

6. M. Nagata, Local rings, Interscience, New York, 1962.

7. J. D. Sally and W. V. Vasconcelos, Flat ideals. I, Comm. Algebra 3 (1975), 531-543.

8. O. Zariski and P. Samuel, Commutative algebra, Van Nostrand, Princeton, N. J., 1958.

Department of Mathematics and Statistics, Case Western Reserve University, Cleveland, Оноо, 44106 